\title{
ON THE MONOTONICITY OF THE PERMANENT
}

\author{
SUK GEUN HWANG
}

(Communicated by Thomas Brylawski)

\begin{abstract}
Let $\Omega_{n}$ denote the set of all $n \times n$ doubly stochastic matrices and let $J_{n}=[1 / n]_{n \times n}$. For $A \in \Omega_{n}$, if $f_{A}(t)=\operatorname{per}\left((1-t) J_{n}+t A\right)$ is a nondecreasing function of $t$ on $[0,1]$, we say that the monotonicity of permanent (abb. MP) holds for $A$. Friedland and Minc [3] proved MP for $\left(n J_{n}-I_{n}\right) /(n-1)$. In [6], Lih and Wang proposed a problem of determining whether MP holds for $J_{n_{1}} \oplus \cdots \oplus J_{n_{k}}, n_{i}>0$.

In this note, we prove MP for $\left(\left(m J_{m}-I_{m}\right) \otimes s J_{s}\right) /(m-1) s$, extending the result of Friedland and Minc, and give an affirmative answer to the Lih and Wang's question.
\end{abstract}

\section{INTRODUCTION}

Let $\Omega_{n}$ denote the set of all $n \times n$ doubly stochastic matrices and let $K_{n}$ denote the $n \times n$ matrix of 1's. As usual, let $I_{n}$ denote the identity matrix of order $n$ and let $J_{n}=K_{n} / n$. For an $n \times n$ matrix $A$ and for a real number $t$, let $A_{t}=(1-t) J_{n}+t A$ and let $f_{A}(t)=\operatorname{per} A_{t}$, the permanent of $A_{t}$.

In [3], Friedland and Minc proved that $f_{A}(t)$ is a monotone increasing function of $t$ on the closed unit interval $[0,1]$ if either $A=I_{n}$ or $A=$ $\left(K_{n}-I_{n}\right) /(n-1)$. The problem of finding matrices $A \in \Omega_{n}$ for which $f_{A}(t)$ is monotone increasing is found in [9, p. 158]. This property of $f_{A}(t)$ now is called the monotonicity of permanent (abb. MP) for $A$. In addition to those matrices which are permutation equivalent to $I_{n}$ or $\left(K_{n}-I_{n}\right) /(n-1)$, several classes of matrices in $\Omega_{n}$ have turned out to satisfy MP (see [5, 8] for example). In [6], Lih and Wang proved MP for $J_{s} \oplus J_{n-s}$ and for

$$
\left[\begin{array}{cc}
0 & Y \\
Y^{T} & Z
\end{array}\right]
$$

where $Y$ is an $(n-t) \times t$ submatrix of $J_{t}$ and $Z=(2-n / t) J_{t}$, and proposed a problem of determining whether MP holds for $J_{n_{1}} \oplus \cdots \oplus J_{n_{k}}$ where $n_{i}>0$, $i=1, \ldots, k$.

Received by the editors May 18, 1988.

1980 Mathematics Subject Classification (1985 Revision). Primary 15A15; Secondary 15A48.

Key words and phrases. Permanent, doubly stochastic matrix, monotonicity.

This research was supported by the Korea Science and Engineering Foundation in 1987. 
In this note we prove MP for $\left(\left(K_{m}-P\right) \otimes K_{s}\right) /(m-1) s$ for any $m \times m$ permutation matrix $P$ where $\otimes$ stands for the Kronecker product and $m \geq 2$, $s \geq 1$, extending Friedland and Minc' result, and give an affirmative answer to Lih and Wang's problem.

For a matrix $A$, let $A(i \mid j)$ denote the matrix obtained from $A$ by deleting the row $i$ and column $j$. Let $D$ be a real valued function of $\Omega_{n}$ defined by

$$
D(A)=\operatorname{per} A-\frac{1}{n^{2}} \sum_{i=1}^{n} \sum_{j=1}^{n} \operatorname{per} A(i \mid j)
$$

for $A \in \Omega_{n}$.

For $A=\left[a_{i j}\right] \in \Omega_{n}$, we compute

$$
\frac{d}{d t} f_{A}(t)=\sum_{i=1}^{n} \sum_{j=1}^{n}\left(a_{i j}-\frac{1}{n}\right) \operatorname{per} A_{t}(i \mid j)
$$

and

$$
D\left(A_{t}\right)=\frac{t}{n} \sum_{i=1}^{n} \sum_{j=1}^{n}\left(a_{i j}-\frac{1}{n}\right) \operatorname{per} A_{t}(i \mid j)
$$

so that

Thus we have

$$
D\left(A_{t}\right)=\frac{t}{n} \frac{d}{d t} f_{A}(t) .
$$

Lemma 1. MP holds for $A \in \Omega_{n}$ if and only if $D\left(A_{t}\right) \geq 0$ for all $t, 0 \leq t \leq 1$.

$$
\text { 2. MP for }\left(\left(K_{m}-I_{m}\right) \otimes K_{s}\right) /(m-1) s \text {. }
$$

To prove MP for $\left(\left(K_{m}-I_{m}\right) \otimes P\right) /(m-1) s$ for an $m \times m$ permutation matrix $P$, we may assume that $P=I_{m}$. Let $n=m s$ so that

$$
\frac{1}{(m-1) s}\left(K_{m}-I_{m}\right) \bigotimes K_{s}=\frac{1}{n-s}\left(K_{n}-K_{s}^{(m)}\right)
$$

where $K_{s}^{(m)}$ denotes the $m$-fold direct sum of $K_{s}$ 's. Note that, in the case of $s=1$,

$$
\frac{1}{n-s}\left(K_{m}-I_{m}\right) \bigotimes K_{s}=\frac{1}{n-1}\left(K_{n}-I_{n}\right) \text {, }
$$

the matrix for which MP was proved by Friedland and Minc [3].

The following Lemma was conjectured by Sinkhorn [10] and proved by Bapat [1].

Lemma 2 [Sinkhorn-Bapat]. Let $A \in \Omega_{n}$ be such that $\operatorname{per} A(i \mid j)=\operatorname{per} A$ for all $i, j=1, \ldots, n$; then either $A=J_{n}$ or $A=\frac{1}{2}\left(I_{n}+P_{n}\right)$, up to permutations of rows and columns where $P_{n}$ stands for the $n \times n$ full-cycle permutation matrix corresponding to the $n$-cycle $(12 \cdots n)$.

Let $A$ be an $n \times n$ real matrix. We say that the positions $(i, j)$ and $(k, l)$ of $A$ are equivalent if there exist permutation matrices $P, Q$ such that $P A Q=A$ 
and the transformation $X \rightarrow P X Q$ takes the $(i, j)$-entry of $X$ onto the $(k, l)$ entry of $P X Q$ for all $n \times n$ matrix $X$.

It is clear that, if the positions $(i, j)$ and $(k, l)$ of $A$ are equivalent then, $\operatorname{per} A(i \mid j)=\operatorname{per} A(k \mid l)$.

Lemma 3. Let $m \geq 2, s \geq 1$ be integers and $n=m s$. For $\left(K_{m}-I_{m}\right) \otimes K_{s}:=$ $\left[x_{i j}\right]$, let $Z=\left\{(i, j) \mid x_{i j}=0\right\}$. Let $A=\left[a_{i j}\right]$ be an $n \times n$ matrix such that all $a_{i j},(i, j) \in Z$, are the same and all $a_{k l},(k, l) \notin Z$, are the same. Then all the $Z$-positions of $A$ are equivalent and all the off $Z$-positions of $A$ are equivalent.

Proof. For $p, q=1, \ldots, m$, let $T_{p q}=\{(i, j) \mid(p-1) s+1 \leq i \leq p s,(q-1) s+$ $1 \leq j \leq q s\}$. Then $Z=\cup_{p=1}^{m} T_{p p}$. Clearly, all the positions of $A$ in a single $T_{p q}$ are equivalent.

To show the equivalence of the position $(1, s+1)$ and $(s+1,1)$ of $A$, we can use the transformation $X \rightarrow P X P$ where

$$
P=\left[\begin{array}{cc}
0 & I_{s} \\
I_{s} & 0
\end{array}\right] \bigoplus I_{n-2 s} .
$$

Thus we see that all the $T_{12} \cup T_{21}$-positions of $A$ are equivalent. In addition to that, this transformation tells us also that all the position of $A$ in $T_{11} \cup T_{12}$ are equivalent.

In the case that $m \geq 3$, we see that the transformation $X \rightarrow Q X Q$ with

$$
Q=I_{s} \bigoplus\left[\begin{array}{cc}
0 & I_{s} \\
I_{s} & 0
\end{array}\right] \bigoplus I_{n-3 s}
$$

assures the equivalence of all the $T_{12} \cup T_{13}$-positions of $A$. Repeating similar arguments a finite number of times, we see that all the $Z$-positions of $A$ are equivalent and all the off $Z$-positions of $A$ are equivalent.

Corollary. Let $m \geq 2, s \geq 1$ be integers, $n=m s$, and let $A=(1 / n-s)\left(K_{m}-\right.$ $\left.I_{m}\right) \otimes K_{s}$. Then, for each $t \in[0,1]$, there exist real numbers $\lambda_{t}, \mu_{t}$ such that

$$
\operatorname{per} A_{t}(i \mid j)-\operatorname{per} A_{t}= \begin{cases}\lambda_{t} & \text { if }(i, j) \in Z \\ \mu_{t} & \text { otherwise. }\end{cases}
$$

Expanding per $A_{t}$ along the first row, we get the following relationship between $\lambda_{t}$ and $\mu_{t}$ :

$$
s(1-t) \lambda_{t}+(n-s)(1-t+n t) \mu_{t}=0 .
$$

Thus, if $t \neq 1$, we have

$$
s(1-t) \lambda_{t}+(n-s+s t) \mu_{t}=0 .
$$

Lemma 4. Let $m \geq 2, s \geq 1$ be integers, $n=m s$, and let $A=(1 / n-s)\left(K_{m}-\right.$ $\left.I_{m}\right) \otimes K_{s}$. Then, for each $t \in[0,1)$,

$$
D\left(A_{t}\right)=\frac{t}{1-t} \mu_{t},
$$


where $\mu_{t}$ is the number defined in (1).

Proof. Taking account of (1), we have

$$
\begin{aligned}
\sum_{i=1}^{n} \sum_{j=1}^{n} \operatorname{per} A_{t}(i \mid j) & =\left(\sum_{(i, j) \in Z}+\sum_{(i, j) \notin Z}\right) \operatorname{per} A_{t}(i \mid j) \\
& =n^{2} \operatorname{per} A_{t}+|Z| \lambda_{t}+\left(n^{2}-|Z|\right) \mu_{t} \\
& =n^{2} \operatorname{per} A_{t}+n s \lambda_{t}+n(n-s) \mu_{t} .
\end{aligned}
$$

Therefore, by (2),

$$
\begin{aligned}
D\left(A_{t}\right) & =\operatorname{per} A_{t}-\frac{1}{n^{2}} \sum_{i=1}^{n} \sum_{j=1}^{n} \operatorname{per} A_{t}(i \mid j) \\
& =-\frac{1}{n}\left(s \lambda_{t}+(n-s) \mu_{t}\right) \\
& =\frac{t}{1-t} \mu_{t} \cdot \quad
\end{aligned}
$$

Now we are ready to prove the following theorem which is an extended version of Friedland and Minc's result [3], of which we give here an elementary combinatorial proof.

Theorem 1. Let $m \geq 2, s \geq 1$ be integers, $n=m s$. Then MP holds for $(1 / n-s)\left(K_{m}-P\right) \otimes K_{s}$ for all $m \times m$ permutation matrices $P$.

Proof. Let $A=(1 / n-s)\left(K_{m}-I_{m}\right) \otimes K_{s}$. To prove the theorem, it suffices to prove the MP for $A$ only. We need to show that $D\left(A_{t}\right) \geq 0$ for all $t \in(0,1)$, or equivalently, that $\mu_{t} \geq 0$ for all $t \in(0,1)$, by Lemmas 1 and 4 .

Suppose that $\mu_{d}<0$ for some $d \in(0,1)$.

Since $J_{n}$ is the unique matrix in $\Omega_{n}$ with the minimum permanent [2], MP holds for matrices in a sufficiently small neighborhood of $J_{n}$ in $\Omega_{n}$. Hence we can take a $c \in(0, d)$ such that $D\left(A_{c}\right)>0$ so that $\mu_{c}>0$. Then, by Mean Value Theorem, there exists a $t \in(c, d)$ such that $\mu_{t}=0$ and hence also that $\lambda_{t}=0$ by (2). But then per $A_{t}(i \mid j)=\operatorname{per} A_{t}$ for all $i, j=1, \ldots, n$, even if $A_{t} \neq J_{n}$ and $A_{t} \neq \frac{1}{2}\left(I_{n}+P_{n}\right)$, contradicting Lemma 2.

Thus we have shown that $\mu_{t}>0$ for all $t \in(0,1)$ and the proof is completed.

\section{MP FOR P.S.D. SYMMETRIC MATRICES IN $\Omega_{n}$}

In this section we give a very simple proof for MP for positive semi-definite (abb. p.s.d.) symmetric doubly stochastic matrices, which assures the MP for $J_{n_{1}} \oplus \cdots \oplus J_{n_{k}}$ where $n_{i}, i=1, \ldots, k$, are positive integers.

The following Lemma can be found in [7].

Lemma 5 [Marcus and Merris]. If $A \in \Omega_{n}$ is p.s.d. symmetric, then $D(A) \geq 0$.

As a corollary of Lemma 5 , we have the following 
Theorem 2. MP holds for any p.s.d. symmetric doubly stochastic matrix.

Proof. If $A \in \Omega_{n}$ is p.s.d. symmetric, then so is $A_{t}$ for all $t \in[0,1]$, so that $D\left(A_{t}\right) \geq 0$ for all $t \in[0,1]$ by Lemma 5 . Now MP for any p.s.d. symmetric doubly stochastic matrix follows from Lemma 1.

Since $J_{n_{1}} \oplus \cdots \oplus J_{n_{k}}, n_{i}>0$, is a p.s.d. symmetric matrix, we have the following result which is the answer to the Lih and Wang's problem in [6].

Corollary 1. MP holds for $J_{n_{1}} \oplus \cdots \oplus J_{n_{k}}, n_{i}>0$.

Corollary 2 ([4]). Let $A_{1}, \ldots, A_{k} \in \Omega_{2}$. Then MP holds for $A_{1} \oplus \cdots \oplus A_{k}$.

Proof. Every matrix in $\Omega_{2}$ is permutation equivalent to a p.s.d. symmetric matrix. Thus, for $A_{1}, \ldots, A_{k} \in \Omega_{2}, A_{1} \oplus \cdots \oplus A_{k}$ is permutation equivalent to a p.s.d. symmetric matrix.

\section{REFERENCES}

1. R. Bapat, Doubly stochastic matrices with equal subpermanents, Linear Algebra Appl. 51 (1983), 1-8.

2. G. P. Egorycev, A solution of van der Waerden's permanent problem, Kirenski Institute of Phisics, Acad. of Sci. SSSR, Preprint IFSO-13M, Kransnoyarsk 1980 (Russian).

3. S. Friedland and H. Minc, Monotonicity of permanents of doubly stochastic matrices, Linear and Multilinear Algebra 6 (1978), 227-231.

4. J. Goldwasser, Contributions to the theory of permanents, Ph.D. Thesis, Univ. of WisconsinMadison, 1983.

5. S. G. Hwang, The monotonicity and the Đokvic conjectures on permanents of doubly stochastic matrices, Linear Algebra Appl. 79 (1986), 127-151.

6. K.-W. Lih and E. T. H. Wang, Monotonicity conjecture on permanents of doubly stochastic matrices, Proc. Amer. Math. Soc. 82 (1981), 173-178.

7. M. Marcus and R. Merris, $A$ relation between permanental and determinantal adjoints, $\mathrm{J}$. Austral. Math. Soc. 15 (1973), 270-271.

8. H. Minc, Theory of permanents 1978-1981, Linear and Multilinear Algebra 12 (1983), 227263.

9. __ Permanents, Encyclopedia of Math. and Its Appl., Addison-Wesley, Reading, Mass., 1978.

10. R. Sinkhorn, Doubly stochastic matrices with dominant p-minors, Linear and Multilinear Algebra 5 (1977), 107-117.

Department of Mathematics, Teachers College, Kyungpook University, Taegu 702-701, KOREA 